\title{
Position-momentum-entangled photon pairs in nonlinear waveguides and transmission lines
}

\author{
Y. Sherkunov, ${ }^{1}$ David M. Whittaker, ${ }^{2}$ and Vladimir Fal' ${ }^{1}{ }^{1}$ \\ ${ }^{1}$ National Graphene Institute, University of Manchester, Manchester M13 9PL, United Kingdom \\ ${ }^{2}$ Department of Physics and Astronomy, University of Sheffield, Sheffield S3 7RH, United Kingdom \\ (Received 27 October 2015; revised manuscript received 22 February 2016; published 25 April 2016)
}

\begin{abstract}
We analyze the correlation properties of light in nonlinear waveguides and transmission lines, predict the position-momentum realization of the Einstein-Podolsky-Rosen paradox for photon pairs in Kerr-type nonlinear photonic circuits, and we show how two-photon entangled states can be generated and detected.
\end{abstract}

DOI: 10.1103/PhysRevA.93.043842

\section{INTRODUCTION}

Most modern communication systems are based on information transfer using light, and quantum properties of light are already being used in securing information transfer protocols. This makes the generation, controlled propagation, and detection of entangled states of photons in optical circuits important elements in communication. Continuous-variable entanglement has been intensively studied in view of developing such protocols [1,2], with the vast majority of works focusing on quadrature components, where entanglement has been observed between the amplitude and phase quadratures of squeezed light [3-6], continuous-variable polarization entanglement [7-9], or transverse position-momentum entanglement in photon pairs produced by spontaneous parametric downconversion processes in crystals [10-13]. However, the implementation of continuous-variable entanglement is mostly limited by free-space optical networks [14] requiring increased complexity, high-precision alignment, and stability.

Here, we propose a theory describing photons entangled over continuous variables in quantum circuits, whose elements are waveguides or chains of high-quality resonators with strong Kerr-type nonlinearity. In such systems the interaction between two photons leads to four-wave mixing [15-20] resulting in the separation of bound pairs of photons, whose propagation in the transmission line is position correlated, from a continuous spectrum of two-photon states. The existence of bound photons discussed in this paper gives ways for a formation of strongly position-momentum-entangled photon states, which are collinear and occupy a single transverse quantized waveguide mode, making them a good candidate for implementation in quantum on-chip systems, in contrast with entangled pairs generated by conventional bulk-crystal entanglers.

The physical system where we expect the entangled photon states to appear include (Sec. II) a Kerr-type nonlinear single-mode waveguide characterized by strong photonphoton coupling [21,22], or (Sec. III) a chain of coupled nonlinear resonators [23-28]. For two photons with momenta $\hbar k_{1}=\hbar\left(k_{0}-\delta k\right)$ and $\hbar k_{2}=\hbar\left(k_{0}+\delta k\right)$ and dispersion

$$
\omega_{k_{0}+\delta k} \approx \omega_{k_{0}}+v \delta k+\beta \delta k^{2} / 2,
$$

where $v$ is the photon group velocity, the variation of the energy of a photon pair

$$
\Delta^{(2)} \omega=\omega_{k_{0}-\delta k}+\omega_{k_{0}+\delta k}-2 \omega_{k_{0}} \approx \beta \delta k^{2} .
$$

As the photon-photon interaction conserves both energy and longitudinal momentum, the two-photon states propagating along the nonlinear transmission line can be described by the Fock function

$$
|\psi\rangle_{2 k_{0}}=\int d k_{1} d k_{2} \delta\left(k_{1}+k_{2}-2 k_{0}\right) f\left(k_{1}-k_{2}\right)\left|k_{1}, k_{2}\right\rangle .
$$

\section{ENTANGLED PHOTON PAIRS IN NONLINEAR WAVEGUIDES}

To demonstrate the principle of position-momentum entanglement of photons in Kerr-nonlinear systems, we first consider the entangled photon pairs in nonlinear optical waveguides. Classically, Kerr nonlinearity in an isotopic medium manifests itself in the third-order polarization

$$
\mathbf{P}^{(3)(+)}=\chi^{(3)}\left[\left(\mathbf{E}^{(-)} \cdot \mathbf{E}^{(+)}\right) \mathbf{E}^{(+)}+\alpha\left(\mathbf{E}^{(+)} \cdot \mathbf{E}^{(+)}\right) \mathbf{E}^{(-)}\right],
$$

where "+" and "-" correspond to positive and negative frequency parts, $\mathbf{E}$ is the electric field, and $\chi^{(3)}$ is the susceptibility of the medium $\chi^{(3)}=\chi_{x y x y}^{(3)}, \alpha=\chi_{x x y y}^{(3)} /\left(2 \chi^{(3)}\right)$. Quantizing electromagnetic field, integrating over transverse degrees of freedom, and neglecting magneto-optical effects ( $\alpha=0$ ) leading to entanglement over polarization degrees of freedom, we arrive at the following Hamiltonian $(\hbar=c=1)$ :

$$
\begin{aligned}
H & =H_{0}+H_{\mathrm{int}}, \quad H_{0}=\sum_{k} \omega_{k} a_{k}^{\dagger} a_{k}, \\
H_{\mathrm{int}} & =\frac{\kappa}{L} \sum_{k_{1}, k_{2}, k_{3}, k_{4}} \delta\left(k_{1}+k_{2}, k_{3}+k_{4}\right) a_{k_{4}}^{\dagger} a_{k_{3}}^{\dagger} a_{k_{1}} a_{k_{2}},
\end{aligned}
$$

where $a_{k}\left(a_{k}^{\dagger}\right)$ is the annihilation (creation) operator of a photon with longitudinal momentum $k$ and energy $\omega_{k}$, and $L$ is the length of the system. The nonlinear term $H_{\text {int }}$ in Eq. (4) describes a photon-photon interaction with coupling $\kappa=\frac{\pi \omega^{2} \chi^{(3)}}{2 n_{r}^{4} A \epsilon_{0}}$, where $n_{r}$ is the refraction index, $A$ is the area occupied by the waveguide mode, and $\epsilon_{0}$ is the vacuum permittivity.

Hamiltonian (4) can be diagonalized exactly in the case of $\Delta^{(2)} \omega \propto \delta k^{2}$ [29]. We consider a sector of the Hilbert space, which consists of all the two-photon states with the total pair momentum $2 k_{0}$ and assume the effective mass approximation for the waveguide dispersion given by $\mathrm{Eq}$. (1). In the coordinate domain, $a_{x}=\frac{1}{\sqrt{L}} \sum_{k} e^{i\left(k-k_{0}\right) x} a_{k}$, the Hamilton Eq. (4) takes the form

$$
\begin{aligned}
H= & \int d x\left(\omega_{k_{0}} a_{x}^{\dagger} a_{x}-i v a_{x}^{\dagger} \partial_{x} a_{x}-\frac{1}{2} \beta a_{x}^{\dagger} \partial_{x}^{2} a_{x}\right) \\
& +\frac{1}{2} \int d x_{1} d x_{2} a_{x_{1}}^{\dagger} a_{x_{2}}^{\dagger} U\left(x_{1}-x_{2}\right) a_{x_{2}} a_{x_{1}}
\end{aligned}
$$


(a)

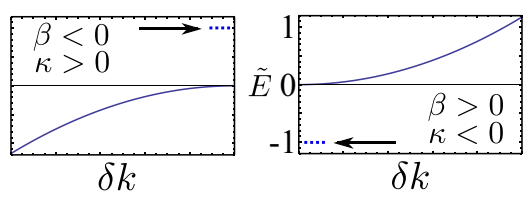

(b)

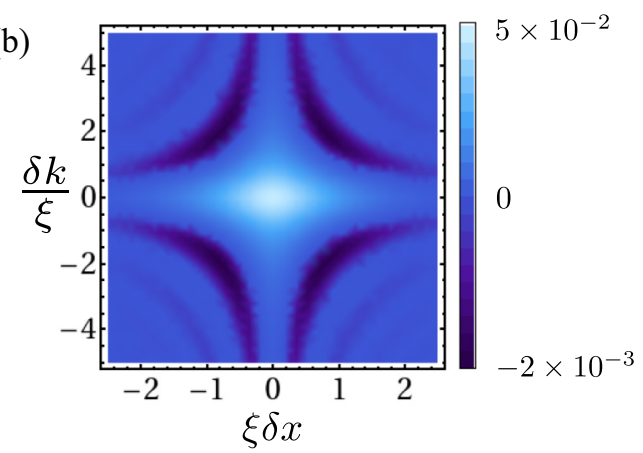

FIG. 1. Entangled two-photon states in nonlinear waveguides. (a) Spectrum of a two-photon state, $\tilde{E}=\left(E-2 \omega_{k_{0}}\right)|\beta| / \kappa^{2}$, with total momentum $2 k_{0}$ in a waveguide with quadratic dispersion (1) for $\beta<0, \kappa>0$ (left) and $\beta>0, \kappa<0$ (right). The solid line corresponds to the continuous spectrum, while the single eigenvalue corresponding to the entangled state is shown by a dashed line. (b) Wigner function of the two-photon entangled state. It takes negative values, which is a hallmark of non-Gaussian entangled states.

where $U\left(x_{1}-x_{2}\right)=2 \kappa \delta\left(x_{1}-x_{2}\right)$. For a two-photon state, described by the wave function

$$
|\psi\rangle=\int d x_{1} d x_{2} f\left(x_{1}, x_{2}\right) a_{x_{1}}^{\dagger} a_{x_{2}}^{\dagger}|0\rangle,
$$

this leads to the following Schrödinger equation:

$$
\begin{aligned}
{\left[2 \omega_{k_{0}}\right.} & \left.-i v\left(\partial_{x_{1}}+\partial_{x_{2}}\right)-\frac{1}{2} \beta\left(\partial_{x_{1}}^{2}+\partial_{x_{2}}^{2}\right)+2 \kappa \delta\left(x_{1}-x_{2}\right)\right] \\
& \times f\left(x_{1}, x_{2}\right)=E f\left(x_{1}, x_{2}\right),
\end{aligned}
$$

where $E$ is the energy of a two-photon state. Equation (6) has scattering-state solutions, which correspond to the continuous spectrum of noninteracting photons with energies given by Eq. (2) [see Fig. 1(a)]. When the curvature of the waveguide dispersion $\beta$ and the photon-photon coupling constant $\kappa$ are of opposite signs, $\beta \kappa<0$, there exists a bound-state solution with

$$
f\left(x_{1}, x_{2}\right)=\sqrt{\frac{\xi}{2 L}} \exp \left[-\left|x_{1}-x_{2}\right| \xi\right], \quad \xi=|\kappa / \beta| .
$$

The energy of this state is split from the continuum of weakly correlated scattering states, as we show in Fig. 1(a), and it is given by

$$
E_{b}=2 \omega_{k_{0}}-\kappa^{2} / \beta
$$

as expected from binding of a one-dimensional massive particle to an attractive $\delta$-functional potential well [30]. In the momentum domain, the two-photon bound-state wave function is given by Eq. (3) with

$$
f\left(k_{1}-k_{2}\right)=\frac{8 \xi^{3 / 2}}{\sqrt{2 L}\left[\left(k_{1}-k_{2}\right)^{2}+4 \xi^{2}\right]} .
$$

The state (9) can be characterized by the Wigner function defined as the expectation value $W\left(x_{1}, k_{1} ; x_{2}, k_{2}\right)=$
$\pi^{-2}\left\langle\psi\left|\Pi\left(x_{1}, k_{1}\right) \otimes \Pi\left(x_{2}, k_{2}\right)\right| \psi\right\rangle$ of the parity operator $\Pi(x, k)=\int d \zeta e^{-2 i x \zeta} a_{k+\zeta}^{\dagger}|0\rangle\langle 0| a_{k-\zeta}$. After straightforward calculations, one can find

$$
\begin{aligned}
W\left(x_{1}, k_{1} ; x_{2}, k_{2}\right)= & \frac{\xi^{2} e^{-2 \xi|\delta x|}}{2 \pi^{2}\left(\delta k^{2}+\xi^{2}\right)} \\
& \times\left(\cos (2 \delta k|\delta x|)+\frac{\xi}{\delta k} \sin (2 \delta k|\delta x|)\right) \\
& \times \delta\left(k_{1}+k_{2} ; 2 k_{0}\right),
\end{aligned}
$$

where $\delta x=x_{1}-x_{2}$. This function is negative for $\cos (2 \delta k|\delta x|)+\xi / \delta k \sin (2 \delta k|\delta x|)<0$, as shown in Fig. 1(b), which implies that the state (9) is entangled in positionmomentum degrees of freedom [31]. Moreover, for $\xi \rightarrow \infty$, the two-photon wave function approaches the ideal EinsteinPodolsky-Rosen (EPR) state (see Appendix A).

Alternatively, to demonstrate that the state (9) is entangled in position-momentum degrees of freedom, one can find the uncertainties $\Delta\left(x_{1}-x_{2}\right)$ and $\Delta\left(k_{1}+k_{2}\right)$ calculated over the joint probability distributions $P\left(x_{1}, x_{2}\right)$ and $P\left(k_{1}, k_{2}\right)$, respectively, for which the separability criterion $[2,32,33]$,

$$
\left[\Delta\left(x_{2}-x_{1}\right)\right]^{2}\left[\Delta\left(k_{2}+k_{1}\right)\right]^{2} \geqslant 1,
$$

can be applied. Although the states for which the inequality (11) is violated are inseparable, they do not necessarily lead to an EPR paradox. In order for an EPR paradox to arise, correlations must violate a more strict inequality [34],

$$
\left[\Delta\left(x_{2}-x_{1}\right)\right]^{2}\left[\Delta\left(k_{2}+k_{1}\right)\right]^{2} \geqslant 1 / 4,
$$

which can be accessible experimentally [13]. Assuming that the system is driven by a Gaussian beam of width $W_{p}$ in momentum space, we find that the entangled photon states are described by the wave function (3) with the $\delta$ function substituted by the Gaussian $\delta\left(k_{1}+k_{2}-2 k_{0}\right) \rightarrow(2 / \pi)^{1 / 4}$ $\left(L / 2 \pi W_{p}\right)^{1 / 2} \exp \left[-\left(k_{1}+k_{2}-2 k_{0}\right)^{2} / W_{p}^{2}\right]$ and $f\left(k_{1}-k_{2}\right)$ given by Eq. (9). For the case of a narrow Gaussian beam with $W_{p} \ll \xi$, we find $\left[\Delta\left(x_{2}-x_{1}\right)\right]^{2}\left[\Delta\left(k_{2}+k_{1}\right)\right]^{2}=\frac{1}{8}\left(\frac{W_{p}}{\xi}\right)^{2}$, which violates both inequalities (11) and (12).

\section{ENTANGLED PHOTON PAIRS IN CHAINS OF NONLINEAR RESONATORS}

Another system, where entangled photon pairs may appear, is a chain (with period $b$ ) of $N$ coupled resonators illustrated in Fig. 2(a). Here, each optical circuit element is characterized by a single-photonic mode of frequency $\omega_{c}$ and a nonlinear on-site photon-photon interaction $u$. The photons can hop between the neighboring cavities with an amplitude $J$, which can be described by the Bose-Hubbard model,

$$
H=\sum_{j}\left[\omega_{c} a_{j}^{\dagger} a_{j}+J\left(a_{j+1}^{\dagger} a_{j}+\text { H.c. }\right)+u a_{j}^{\dagger} a_{j}^{\dagger} a_{j} a_{j}\right],
$$

where $a_{j}\left(a_{j}^{\dagger}\right)$ is the annihilation (creation) operator of a photon on site $j$. Hamiltonian (13) can also be diagonalized exactly in the two-particle subspace of the Hilbert space $[35,36]$. Using the periodic boundary conditions for a closed chain $(j=N+$ $1 \equiv 1)$, the system is described by the following single-photon 
(a)

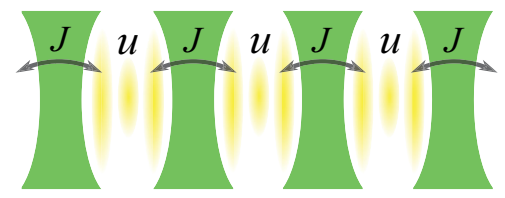

(b)
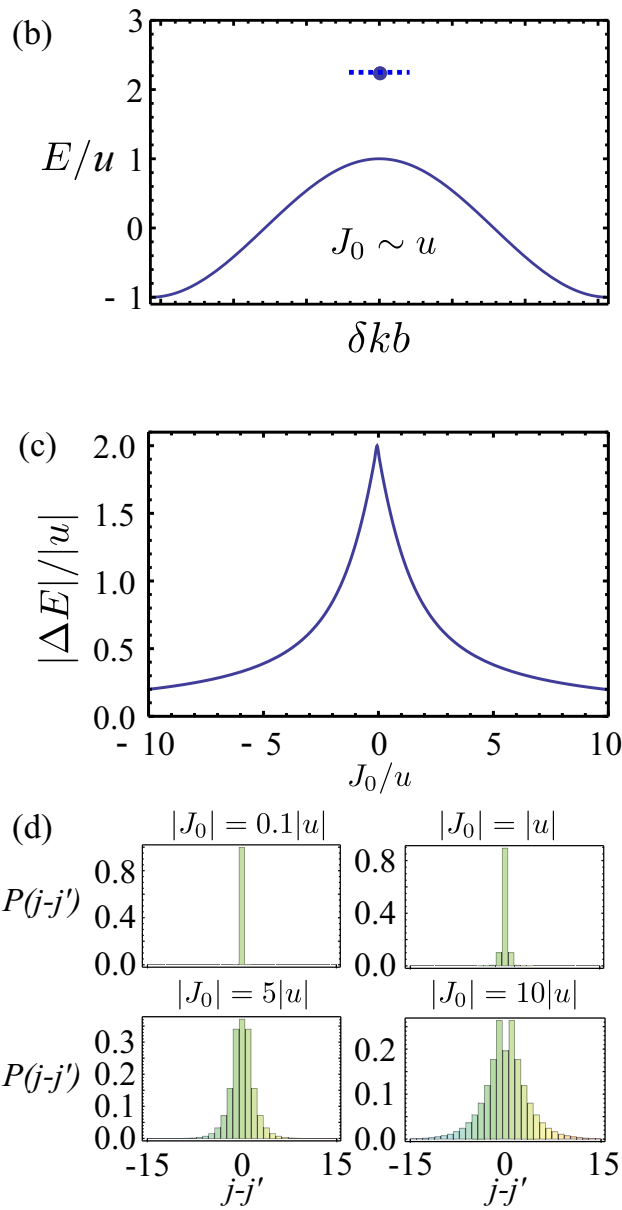

FIG. 2. Entangled photon pairs in chains of coupled nonlinear resonators. (a) Schematic of the setup. (b) An example of a twophoton spectrum for $J_{0} \sim u$ and $\omega_{c}=0$. (c) Split of the boundstate eigenvalue from the spectrum edge $\Delta E$ as a function of $J_{0}=4 J \cos \left(k_{0} a\right)$. (d) Joint probability distribution $P$ for various ratios $J_{0} / u$.

dispersion $\omega_{k}$,

$$
\begin{aligned}
\omega_{k} & =\omega_{c}+2 J \cos (k b), \\
k & =2 \pi n /(N b), \quad n=0,1,2, \ldots, N-1 .
\end{aligned}
$$

For the two-photon states,

$$
|\psi\rangle=\sum_{j^{\prime} \geqslant j} f\left(j^{\prime}-j\right) e^{i k_{0} b\left(j+j^{\prime}\right)} a_{j}^{\dagger} a_{j^{\prime}}^{\dagger}|0\rangle,
$$

the Schrödinger equation $H|\psi\rangle=E|\psi\rangle$ is equivalent to

$$
\begin{aligned}
J_{0} f(1) & =2\left(E-2 \omega_{c}-2 u\right) f(0), \\
J_{0} f(j+1) & =2\left(E-2 \omega_{c}\right) f(j)-\left(1+\delta_{j, 1}\right) J_{0} f(j-1) .
\end{aligned}
$$

Here, $J_{0}=4 J \cos \left(k_{0} b\right)$ is the energy of two noninteracting photons, each with quasimomentum $k_{0}$.

The scattering-state solution has the energy of the noninteracting photon pair $E_{\mathrm{sc}}=2 \omega_{c}+J_{0} \cos (\delta k b)$ and wave function

$$
f_{\mathrm{sc}}(j)=2\left(\cos (\delta k j b)-\frac{2 u \sin (\delta k j b)}{J_{0} \sin (\delta k b)}\right) f(0) .
$$

Moreover, Eq. (15) has a bound-state solution independent of the signs of the coupling constant $u$ and curvature of the spectrum. This state has energy

$$
E_{b}=2 \omega_{c}+\operatorname{sgn}(u) \sqrt{J_{0}^{2}+4 u^{2}}
$$

and wave function

$$
\begin{aligned}
f_{b}(j) & =2 \sqrt{\frac{\left(1-\eta^{2}\right)}{N\left(1+3 \eta^{2}\right)}}\left(\eta^{|j|}-\frac{\delta_{j, 0}}{2}\right), \\
\eta & =\frac{1}{J_{0}}\left(-2 u+\operatorname{sgn}(u) \sqrt{J_{0}^{2}+4 u^{2}}\right) .
\end{aligned}
$$

In the wave-number representation, this reads

$$
|\psi\rangle=\sqrt{\frac{\left(1-\eta^{2}\right)^{3}}{N\left(1+3 \eta^{2}\right)}} \sum_{k_{1} k_{2}} \frac{\delta\left(k_{1}+k_{2} ; 2 k_{0}\right) a_{k_{1}}^{\dagger} a_{k_{2}}^{\dagger}}{1-2 \eta \cos \left[\frac{\left(k_{1}-k_{2}\right) b}{2}\right]+\eta^{2}}|0\rangle .
$$

This state is separated from the quasicontinuum of scattering states as we show in Fig. 2(b).

For strong photon-photon coupling $|u| \gg\left|J_{0}\right|$, Eq. (17) yields

$$
\begin{aligned}
f_{b}(j) & =\frac{2}{\sqrt{N}}\left(1-\frac{\delta_{j, 0}}{2}\right)\left(\frac{J_{0}}{4 u}\right)^{|j|}, \\
E_{b} & =2 \omega_{c}+2 u+J_{0}^{2} /(4 u) .
\end{aligned}
$$

In this case, the photon-photon correlation length is small, so the two correlated photons tend to occupy the same resonator, with their energy approaching the on-site interaction energy $2 u$ independent of whether the interaction is repulsive or attractive [see Figs. 2(c) and 2(d)]. It is worth mentioning that, for $\eta \rightarrow 0$, the wave function (18) mimics a perfect EPR pair (see Appendix A).

In the case of weak photon-photon coupling $\left|J_{0}\right| \gg|u|$, the correlated photon pair has a large correlation radius and a small energy separation from the continuum of scattering states [Figs. 2(c) and 2(d)]. In this case we find

$$
f_{b}(j)=\frac{2}{\sqrt{N}}\left(1-\frac{\delta_{j, 0}}{2}\right)\left[\operatorname{sgn}\left(u J_{0}\right)\left(1-\frac{|2 u|}{\left|J_{0}\right|}+\frac{2 u^{2}}{J_{0}^{2}}\right)\right]^{|j|}
$$

and

$$
E_{b}=2 \omega_{c}+\operatorname{sgn}(u)\left(\left|J_{0}\right|+2 u^{2} /\left|J_{0}\right|^{2}\right),
$$

in agreement with the continuous model Eq. (7) [37]. The transition between the two extremes of strongly and weakly interacting photons is shown in Fig. 2(d).

Experimentally, the entangled states discussed above could be generated by applying a coherent pump, such as a monochromatic laser beam, to the chain of resonators. The results of numerical simulations of the generation of entangled 

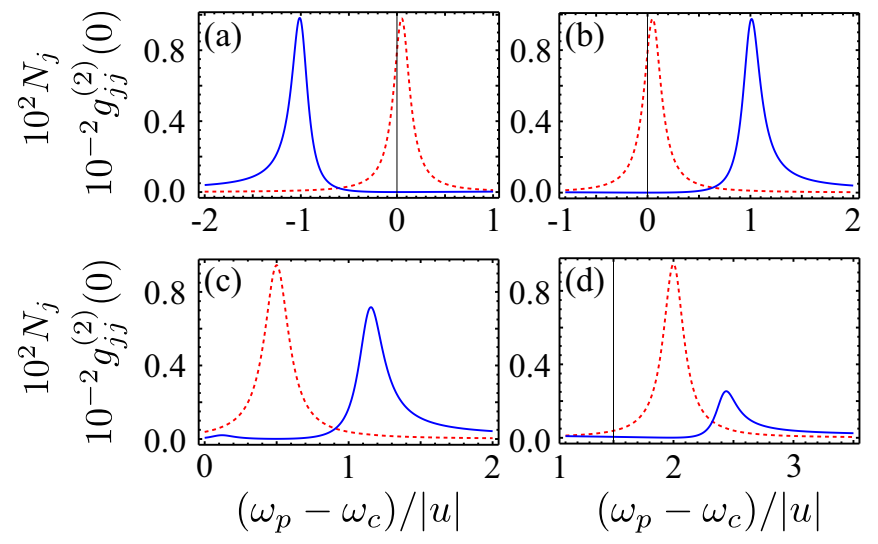

FIG. 3. Steady-state average number of photons $N_{j}$ (dashed line) and on-site zero-time-delay correlation function $g_{j j}^{(2)}(0)$ (solid line) in resonator $j$, as a function of pumping frequency $\omega_{p}$, for a coherently pumped short chain (three resonators) with pumping amplitude $F=$ $10^{-2}|u|$, photon decay rate $\gamma=0.1|u|$, and pair momentum $2 k_{0}=0$. (a) $J_{0}=0.1|u|, u<0$; (b) $J_{0}=0.1|u|, u>0$; (c) $J_{0}=|u|, u>0$; (d) $J_{0}=4|u|, u>0$. Note that far from the resonances, $g_{j j}^{(2)}(0) \rightarrow 1$.

photon states in a closed chain of three lossy cavities driven by a weak coherent laser source shown in Fig. 3 (see Appendix B) suggest that the most effective generation of two-photon entangled states occurs when the pump frequency $\omega_{p}$ satisfies the resonant condition for bound-photon pairs, $\omega_{p}=E_{b} / 2$, for which the zero-time-delay on-site correlation function $g_{j j}^{(2)}=$ $\left\langle\left(a_{j}^{\dagger}\right)^{2} a_{j}^{2}\right\rangle /\left\langle a_{j}^{\dagger} a_{j}\right\rangle^{2}$ takes its maximum value [38,39]. Singlephoton resonance takes place at $\omega_{p}=\omega_{c}+J_{0} / 2$, which can be seen as a maximum of on-site number of photons $N_{j}$ and minimum of $g_{j j}^{(2)}$. This corresponds to the generation of scattering states. The momentum of the two-photon state $2 k_{0}$ is determined by the site-dependent phase of pumping.

\section{CONCLUSIONS}

To conclude, we have shown that photon pairs entangled over continuous variables such as position and momentum can be generated in quantum systems whose elements are either nonlinear waveguides or chains of optical or microwave nonlinear resonators due to a photon-photon interaction stemmed by Kerr-type nonlinearity. In the case of a strong photon-photon interaction, the generated states give a good approximation to the EPR state.

The theory is formulated independently of the frequency range of photons used. It can be applied to visible-range polaritonic waveguide systems, where the nonlinearity is due to an exciton-exciton interaction [40]. It is also applicable to microwave-frequency superconducting transmission lines of high-quality resonators coupled to qubits [41]. The latter system is more appealing because of the controllability of its parameters, high $Q$ factor (low losses), and a stronger nonlinearity [41], which enables one to reach the regime with $u / \gamma \gg 1$, making the effect of losses on the EPR correlations negligible. Strong nonlinearity and low losses can also be achieved in the systems, where atoms in an electromagnetically induced transparency regime $[42,43]$ are coupled to microcavities with high-quality factors $Q$, such as toroidal $\left(Q>10^{8}\right)$ [44] or microrod $\left(Q>10^{9}\right)$ [45] resonators. In these systems, the strength of nonlinearity can reach $u \sim 1.25 \times 10^{7} \mathrm{~s}^{-1}$, while the losses can be as low as $\gamma \sim 2 \times 10^{5} \mathrm{~s}^{-1}[43,44,46,47]$, hence reaching the desirable regime.

The position-momentum-entangled pairs discussed in this paper, in comparison with the ones generated by conventional bulk-crystal entanglers, are collinear and predominantly occupy a single transverse quantized waveguide mode, which offers a potential for the implementation in quantum onchip circuits. Experimentally, the entangled states could be generated by applying coherent pumping to the system with frequency satisfying the resonance condition for boundphoton pairs. These states could be accessed by measuring the two-photon Wigner function in a Hong-Ou-Mandel-type experiment [31]. It can play a role of an entanglement witness taking negative values, as shown in Fig. 1(b), for non-Gaussian entangled states. We have also demonstrated that EPR correlations of the states discussed in this paper would lead to a violation of experimentally accessible [13] criterion (12).

\section{ACKNOWLEDGMENTS}

We thank D. Krizhanovskii, E. Cancellieri, and M. Skolnick for useful discussions. This work was supported by EPSRC Programme Grant No. EP/J007544.

\section{APPENDIX A}

In the case of the waveguide with linear dispersion $(\beta=0)$, one can find $f\left(k_{1}-k_{2}\right)=$ const. This is the ideal positionmomentum-entangled state proposed by Einstein, Podolsky, and Rosen (EPR) [48], in which position $x$ and momenta are perfectly (anti)correlated:

$$
|\psi\rangle=\int d(\delta k)\left|k_{0}+\delta k, k_{0}-\delta k\right\rangle=\int d x e^{2 i k_{0} x}|x, x\rangle .
$$

To demonstrate this, we rewrite the Hamiltonian (4) as an $(N+1) / 2 \times(N+1) / 2$ matrix

$$
H=2 \omega_{k_{0}} \mathbb{I}+\frac{2 \kappa}{L}\left(\begin{array}{cccc}
1 & \sqrt{2} & \sqrt{2} & \ldots \\
\sqrt{2} & 2 & 2 & \ldots \\
\sqrt{2} & 2 & 2 & \ldots \\
\cdots & \ldots & \ldots & \ldots
\end{array}\right)
$$

in the basis spanning $(N+1) / 2$ two-photon states with total momentum $2 k_{0}$. It can be diagonalized exactly, yielding the following eigenvalues: bound-state eigenvalue $E_{b}=2 \omega_{k_{0}}+$ $\kappa k_{\max } / \pi$, where $k_{\max }$ is the maximum wave number corresponding to the breakdown of the linear approximation, and continuous spectrum eigenvalues $E_{\delta k}=2 \omega_{k_{0}}$. The wave functions corresponding to the bound-state wave function are found to be $\psi \propto \frac{1}{\sqrt{2}}\left(a_{k_{0}}^{\dagger}\right)^{2}|0\rangle+\sum_{\delta k>0} a_{k_{0}+\delta k}^{\dagger} a_{k_{0}-\delta k}^{\dagger}|0\rangle$ and the continuous spectrum wave functions are $\psi_{\delta k} \propto-\sqrt{2}\left(a_{k_{0}}^{\dagger}\right)^{2}|0\rangle+$ $a_{k_{0}+\delta k}^{\dagger} a_{k_{0}-\delta k}^{\dagger}|0\rangle$. In the coordinate domain, the bound-state two-photon wave function is $\psi \propto \int d x_{1} d x_{2} e^{i k_{0}\left(x_{1}+x_{2}\right)} \delta\left(x_{1}-\right.$ $\left.x_{2}\right) a_{x_{1}}^{\dagger} a_{x_{2}}^{\dagger}|0\rangle$. 


\section{APPENDIX B}

The density matrix $\rho$, describing the evolution of photons in three coherently driven lossy cavities, obeys the master equation

$\partial_{t} \rho=-i\left[H+H_{p}, \rho\right]+\gamma \sum_{j=1,2,3}\left(2 a_{j} \rho a_{j}^{\dagger}-a_{j}^{\dagger} a_{j} \rho-\rho a_{j}^{\dagger} a_{j}\right)$,

where $\gamma$ is the photon decay rate, $H_{p}=\sum_{j}\left[F_{j}(t) a_{j}^{\dagger}+\right.$ H.c.], and $F_{j}(t)=F e^{-i \omega_{p} t+i \psi_{j}}$ describes coherent pumping with amplitude $F$, frequency $\omega_{p}$, and phase $\psi_{j}$. The latter determines the momentum of generated photons. By finding the steady-state solution of the master equation (B1) for the density matrix determined in the Fock space of photon states with different occupation numbers of the three cavities, $\rho=$ $\sum P\left(m_{1}, m_{2}, m_{3}, n_{1}, n_{2}, n_{3}\right)\left|m_{1}, m_{2}, m_{3}\right\rangle\left\langle n_{1}, n_{2}, n_{3}\right|$, we evaluate the numbers $N_{j}$ of photons in each cavity as well as the zero-time-delay on-site pair correlation function $g_{j j}^{(2)}(0)$. We assumed $\psi_{j}=0$.
[1] S. L. Braunstein and P. van Loock, Rev. Mod. Phys. 77, 513 (2005).

[2] M. D. Reid, P. D. Drummond, W. P. Bowen, E. G. Cavalcanti, P. K. Lam, H. A. Bachor, U. L. Andersen, and G. Leuchs, Rev. Mod. Phys. 81, 1727 (2009).

[3] Z. Y. Ou, S. F. Pereira, H. J. Kimble, and K. C. Peng, Phys. Rev. Lett. 68, 3663 (1992).

[4] C. Silberhorn, P. K. Lam, O. Weiß, F. König, N. Korolkova, and G. Leuchs, Phys. Rev. Lett. 86, 4267 (2001).

[5] W. P. Bowen, R. Schnabel, P. K. Lam, and T. C. Ralph, Phys. Rev. Lett. 90, 043601 (2003).

[6] A. S. Villar, L. S. Cruz, K. N. Cassemiro, M. Martinelli, and P. Nussenzveig, Phys. Rev. Lett. 95, 243603 (2005).

[7] W. P. Bowen, N. Treps, R. Schnabel, and P. K. Lam, Phys. Rev. Lett. 89, 253601 (2002).

[8] O. Gloeckl, J. Heersink, N. Korolkova, G. Leuchs, and S. Lorenz, J. Opt. B 5, S492 (2003).

[9] R. Dong, J. Heersink, J.-I. Yoshikawa, O. Gloeckl, U. L. Andersen, and G. Leuchs, New J. Phys. 9, 410 (2007).

[10] J. C. Howell, R. S. Bennink, S. J. Bentley, and R. W. Boyd, Phys. Rev. Lett. 92, 210403 (2004).

[11] M. D’Angelo, Y.-H. Kim, S. P. Kulik, and Y. Shih, Phys. Rev. Lett. 92, 233601 (2004).

[12] M. Edgar, D. Tasca, F. Izdebski, R. Warburton, J. Leach, M. Agnew, G. Buller, R. Boyd, and M. Padgett, Nat. Commun. 3, 984 (2012).

[13] P.-A. Moreau, F. Devaux, and E. Lantz, Phys. Rev. Lett. 113, 160401 (2014)

[14] G. Masada, K. Miyata, A. Politi, T. Hashimoto, J. L. O’Brien, and A. Furusawa, Nat. Photonics 9, 316 (2015).

[15] L. J. Wang, C. K. Hong, and S. R. Friberg, J. Opt. B 3, 346 (2001).

[16] J. G. Rarity, J. Fulconis, J. Duligall, W. J. Wadsworth, and P. S. J. Russell, Opt. Express 13, 534 (2005).

[17] X. Li, P. L. Voss, J. E. Sharping, and P. Kumar, Phys. Rev. Lett. 94, 053601 (2005).

[18] J. E. Sharping, K. F. Lee, M. A. Foster, A. C. Turner, B. S. Schmidt, M. Lipson, A. L. Gaeta, and P. Kumar, Opt. Express 14, 12388 (2006).

[19] E. Engin, D. Bonneau, C. M. Natarajan, A. S. Clark, M. G. Tanner, R. H. Hadfield, S. N. Dorenbos, V. Zwiller, K. Ohira, N. Suzuki et al., Opt. Express 21, 27826 (2013).

[20] I. Carusotto and G. C. La Rocca, Phys. Rev. B 60, 4907 (1999).

[21] P. M. Walker, L. Tinkler, M. Durska, D. M. Whittaker, I. J. Luxmoore, B. Royall, D. N. Krizhanovskii, M. S. Skolnick,
I. Farrer, and D. A. Ritchie, Appl. Phys. Lett. 102, 012109 (2013).

[22] P. M. Walker, L. Tinkler, D. V. Skryabin, A. Yulin, B. Royall, I. Farrer, D. A. Ritchie, M. S. Skolnick, and D. N. Krizhanovskii, Nat. Commun. 6, 8317 (2015).

[23] A. Wallraff, D. I. Schuster, A. Blais, L. Frunzio, R.-S. Huang, J. Majer, S. Kumar, S. M. Girvin, and R. J. Schoelkopf, Nature (London) 431, 162 (2004).

[24] D. I. Schuster, A. A. Houck, J. A. Schreier, A. Wallraff, J. M. Gambetta, A. Blais, L. Frunzio, J. Majer, B. Johnson, M. H. Devoret et al., Nature (London) 445, 515 (2007).

[25] M. Hofheinz, E. M. Weig, M. Ansmann, R. C. Bialczak, E. Lucero, M. Neeley, A. D. O'Connell, H. Wang, J. M. Martinis, and A. N. Cleland, Nature (London) 454, 310 (2008).

[26] M. H. Devoret and R. J. Schoelkopf, Science 339, 1169 (2013).

[27] Y. Yin, Y. Chen, D. Sank, P. J. J. O’Malley, T. C. White, R. Barends, J. Kelly, E. Lucero, M. Mariantoni, A. Megrant et al., Phys. Rev. Lett. 110, 107001 (2013).

[28] R. Barends, J. Kelly, A. Megrant, A. Veitia, D. Sank, E. Jeffrey, T. C. White, J. Mutus, A. G. Fowler, B. Campbell et al., Nature (London) 508, 500 (2014).

[29] H. B. Thacker, Rev. Mod. Phys. 53, 253 (1981).

[30] L. Landau and E. Lifshitz, Quantum Mechanics: Nonrelativistic Theory (Butterworth-Heinemann, Oxford, U.K., 1977).

[31] T. Douce, A. Eckstein, S. P. Walborn, A. Z. Khoury, S. Ducci, A. Keller, T. Coudreau, and P. Milman, Sci. Rep. 3, 3530 (2013).

[32] S. Mancini, V. Giovannetti, D. Vitali, and P. Tombesi, Phys. Rev. Lett. 88, 120401 (2002).

[33] L.-M. Duan, G. Giedke, J. I. Cirac, and P. Zoller, Phys. Rev. Lett. 84, 2722 (2000).

[34] M. D. Reid, Phys. Rev. A 40, 913 (1989).

[35] M. Valiente and D. Petrosyan, J. Phys. B 41, 161002 (2008).

[36] M. Lewenstein, A. Sanpera, and V. Ahufinger, Ultracold Atoms in Optical Lattices: Simulating Quantum Many-Body Systems (Oxford University Press, Oxford, U.K., 2012).

[37] Indeed, taking $N \rightarrow \infty$ and expanding Eq. (14) in the vicinity of $k \approx k_{0}$ up to the second order in $\delta k$, one arrives at Eq. (1) with $\omega_{k_{0}}=J_{0} / 2, v=2 J b \sin \left(k_{0} b\right)$, and $\beta=-J_{0} b^{2} / 2$. Substituting these results into Eqs. (7) and (8), taking into account that for $N \rightarrow \infty$ the coupling constants of both models are related as $\kappa=u b$, and expanding up to the second order in $u / J_{0}$, we arrive at Eqs. (19) and (20).

[38] Y. Sherkunov, D. M. Whittaker, H. Schomerus, and V. Fal'ko, Phys. Rev. A 90, 033845 (2014).

[39] Y. Sherkunov, D. M. Whittaker, and V. Fal'ko, Phys. Rev. A 93, 023843 (2016). 
[40] V. Timofeev and D. Sanvitto, Exciton Polaritons in Microcavities (Springer, Berlin, 2014).

[41] A. A. Houck, H. E. Tureci, and J. Koch, Nat. Phys. 8, 292 (2012).

[42] A. Imamoḡlu, H. Schmidt, G. Woods, and M. Deutsch, Phys. Rev. Lett. 79, 1467 (1997).

[43] M. J. Hartmann and M. B. Plenio, Phys. Rev. Lett. 99, 103601 (2007).

[44] D. K. Armani, T. J. Kippenberg, S. M. Spillane, and K. J. Vahala, Nature (London) 421, 925 (2003).
[45] P. Del'Haye, S. A. Diddams, and S. B. Papp, Appl. Phys. Lett. 102, 221119 (2013).

[46] T. Aoki, B. Dayan, E. Wilcut, W. P. Bowen, A. S. Parkins, T. J. Kippenberg, K. J. Vahala, and H. J. Kimble, Nature (London) 443, 671 (2006).

[47] S. M. Spillane, T. J. Kippenberg, K. J. Vahala, K. W. Goh, E. Wilcut, and H. J. Kimble, Phys. Rev. A 71, 013817 (2005).

[48] A. Einstein, B. Podolsky, and N. Rosen, Phys. Rev. 47, 777 (1935). 